\title{
Interleukin-12 in patients with cancer is synthesized by peripheral helper $\mathrm{T}$ lymphocytes
}

\author{
MARCIA A. MICHELIN ${ }^{1,2}$, LETICIA MONTES ${ }^{1}$, ROSEKEILA S. NOMELINI ${ }^{1,3}$, \\ DOUGLAS R. ABDALLA ${ }^{1}$, ANDRE A. R. ALEIXO ${ }^{1}$ and EDDIE F. C. MURTA ${ }^{1,3}$ \\ ${ }^{1}$ Oncology Research Institute (IPON); Disciplines of ${ }^{2}$ Immunology and ${ }^{3}$ Gynecology and Obstetrics, \\ Federal University of Triângulo Mineiro, Uberaba, Minas Gerais 38025-440, Brazil
}

Received August 26, 2014; Accepted May 12, 2015

DOI: $10.3892 / \mathrm{ol} .2015 .3470$

\begin{abstract}
The production of cytokines by helper T lymphocytes is a critical event in the immune response, as alterations in the regulation of this process may result in an appropriate immune response, persistent infection or the development of autoimmune disease. Previously, this group has used flow cytometry to demonstrate the expression of interleukin-12 (IL-12) in peripheral blood CD4+ T lymphocytes from patients and mice with advanced cancer. The aim of the present study was to investigate whether CD4+ T lymphocytes from the peripheral blood (PB) of patients with cancer produce IL-12, using molecular approaches, flow cytometry and cellular imaging techniques. CD3+ and CD4+ cells, and cells producing IL-12, were isolated from the PB obtained from patients with cancer, using a cell sorting flow cytometry technique. The positivity of cells for CD3, CD4 and IL-12, which were identified by cell sorting, was visualized using immunofluorescent cellular imaging. Total RNA was extracted from the CD3+CD4+IL-12+ cells, obtained by cell sorting, for confirmation of the presence of IL-12 mRNA, using reverse transcription-polymerase chain reaction (RT-PCR). RT-PCR demonstrated the presence of IL-12 mRNA in all patients $(n=14)$, in contrast to the control group, in whom IL-12 expression was not detected. Immunofluorescent analysis of CD4+ T lymphocytes showed positive intracytoplasmatic IL-12 staining. These results demonstrated that $\mathrm{CD} 3+\mathrm{CD} 4+\mathrm{T}$ lymphocytes in the PB of patients with cancer have the capacity to synthesize and express IL-12.
\end{abstract}

Correspondence to: Professor Márcia A. Michelin, Discipline of Immunology, Federal University of Triângulo Mineiro, 1940 Avenida Getulio Guarita, Uberaba, Minas Gerais 38022-200, Brazil E-mail: michelinimuno@icbn.uftm.edu.br

Key words: CD4+ $\mathrm{T}$ lymphocytes, interleukin-12, cytokine, peripheral blood, production

\section{Introduction}

One of the most significant findings in the field of immunology was the identification by Mosmann et al (1) in 1989, of different types of CD4+ T lymphocytes, termed Th1 and Th2, which each have the capacity to synthesize specific cytokines. These different clones of helper lymphocytes are important for the production of an efficient immune response during distinct conditions, leading to the activation of different immune cell types in particular situations, including viral, bacterial or fungal infections. An imbalance of these clones may lead to the development of certain diseases, for example, autoimmune disease and cancer.

A number of studies have investigated these subtypes of helper T lymphocytes. CD4+ T lymphocytes are characterized as cells of the adaptive immune response that produce multiple cytokines and are divided into subtypes, according to their production of particular transcription factors, as Th1, Th2, Th9, Th17 or Treg. Of the numerous cytokines produced by the immune system, interleukin-12 (IL-12) is involved in the differentiation of Th1 phenotypes, is important for mounting antitumor responses (2) and possesses unique and distinctive features such as activation of cellular responses (3).

A previous study demonstrated that IL-12 is produced by dendritic cells (DC), macrophages and human B-lymphoblastoid cells (4). In addition, this group has previously shown that patients with cancer who were administered an autologous DC vaccine, demonstrated a stimulation of IL-12 expression by CD4+ T lymphocytes (5). IL-12 had been intracellularly marked, with the aim of investigating this cytokine following DC vaccination. When peripheral lymphocytes from patients with cancer were analyzed by flow cytometry, IL-12 expression was observed in CD3+CD4+ T lymphocytes.

Following these initial results, our group conducted a further study in order to confirm IL-12 expression in this cell population, using double staining of CD3+CD4+ T lymphocytes (6). Cultured peripheral blood (PB) cells of healthy donors exhibited no increase in IL-12 expression following lipopolysaccharide and phytohemagglutinin stimulation, compared with those of the control group. By contrast, the percentage of CD3+CD4+IL12+cells collected from the PB of patients with cancer, were significantly increased compared with those from healthy controls. IL-12 expression 
by CD4+ T cells was also observed in splenocytes from mice with 7,12-dimethyl-benzanthracene-induced breast tumors (6).

These results suggested that CD4+ T Lymphocytes obtained from humans and mice with cancer express intracellular IL-12 protein. Therefore, the aim of the present study was to investigate whether CD4+ T Lymphocytes collected from the PB of patients with cancer produce IL-12, using molecular approaches and microscopy.

\section{Patients and methods}

Patients. PB samples from patients and controls were collected at the Clinical Hospital of the Federal University of Triângulo Mineiro (Uberaba, Brazil) by intravenous puncture from 14 women with cancer (age range, 38 to 72 years; median, 56 years), who had not received treatment or who were $\geq 2$ months post-treatment. The sites and stages of cancer were as follows: Endometrial (adenocarcinoma), stage IA $(n=1)$, stage IB $(n=1)$ and stage IIIC $(n=1)$; ovarian, abdominal recidivate of granulosa cell ovarian tumor $(n=1)$, stage IIIC $\mathrm{n}=1)$ and stage IV epithelial carcinoma $(\mathrm{n}=1)$; uterine cervical, stage IB2 $(n=1)$, stage IIB $(n=2)$, and stage IIIB $(n=3)$, of which all cases were epidermoid, with the exception of the one patient with adenocarcinoma at stage IIIB; vulval (epidermoid; stage I; $n=1)$; and diffuse non Hodgkin's Lymphoma following invasive ductal breast cancer treatment $(n=1)$. Control samples were obtained from six healthy donors.

All the patients and healthy controls involved in the present study were counselled regarding its aims, and provided written consent for participation. The study was approved by the Ethics committee of the Federal University of The Triângulo Mineiro Uberaba (record no. 683-2006).

Flow cytometry. PB samples were evaluated using a BD FACSAria III ${ }^{\mathrm{TM}}$ flow cytometer and cell sorter (BD Biosciences, San Diego, CA, USA), according to the manufacturer's instructions, with specific monoclonal antibodies for each marker. Briefly, leukocytes were isolated from peripheral blood samples and red cells were lysed using a standard cell lysing protocol (BD Biosciences-FACS ${ }^{\mathrm{TM}}$ Lysing Solution; BD Biosciences) for $10 \mathrm{~min}$. Cells were then centrifuged at $290 \mathrm{x} \mathrm{g}$ for $10 \mathrm{~min}$ at $4^{\circ} \mathrm{C}$. Cells were washed 3 times by centrifugation at $290 \mathrm{x}$ g for $10 \mathrm{~min}$ with PBS, and the cell precipitate was added to $2 \mu \mathrm{l}$ of protein transfer inhibitor (BD Golgistop ${ }^{\mathrm{TM}}$; BD Biosciences) and incubated for $20 \mathrm{~min}$ at $4^{\circ} \mathrm{C}$, prior to washing by centrifugation at $290 \mathrm{x}$ g for $10 \mathrm{~min}$ with PBS in order to remove excess inhibitor.

Cells were transferred to test tubes in order to perform extracellular labelling. The tubes were divided into control isotypes and test groups, to enable the identification of total T (CD3+) lymphocytes, $\mathrm{T}$ helper (CD4+) lymphocytes and macrophages (CD14+), with CD14+ cells identified in order to test the hypothesis that only CD3+CD4+cells were present in the selected gate. All antibodies (BD Biosciences) were monoclonal and specific for the markers studied, as follows: Fluorescein isothiocyanate (FITC)-labeled mouse anti-human CD3 (catalog no. 555339; dilution, 1:200), allophycocyanin-labeled mouse anti-human CD4 (catalog no. 555349; dilution, 1:200), and FITC-labeled mouse anti-human CD14 (catalog no. 555397; dilution, 1:200). Once extracellular tagging was complete, cells were incubated at $4^{\circ} \mathrm{C}$ for $30 \mathrm{~min}$ and rinsed twice by centrifugation at $290 \mathrm{x} \mathrm{g}$ for 10 min with PBS.

Subsequently, a fixation and permeabilization solution was added (BD Cytofix/Cytoperm ${ }^{\mathrm{TM}}$ ) for $20 \mathrm{~min}$ at $4^{\circ} \mathrm{C}$. The cells were rinsed twice again with Perm/wash buffer (BD Biosciences) prior to the second tagging. For intracellular identification, cells were incubated with a monoclonal phycoerythrin-labeled mouse $\operatorname{IgG}_{1}$ anti-human antibody against the p40 subunit of IL-12 (catalog no. 559329; BD Biosciences) at $4^{\circ} \mathrm{C}$ for $30 \mathrm{~min}$. Finally, cells were re-suspended in $500 \mu \mathrm{l}$ of PBS for cytometric analysis, using BD FACSAria III. CD3+CD4+IL-12+ cells were identified and submitted to separation using the cell sorter. A proportion of the cells obtained following sorting were used for RT-PCR analysis, while the remaining cells were subjected to image analysis.

RT-PCR analysis. CD3+CD4+IL-12+ cells were subjected to total RNA extraction, using TRIzol ${ }^{\mathrm{TM}}$ (Invitrogen Life Technologies, Carlsbad, CA, USA), according to the manufacturer's instructions, and synthesis of cDNA was subsequently performed. Briefly, dried RNA was resuspended in $11.0 \mu \mathrm{l}$ ultrapure water treated with diethylpyrocarbonate (DEPC; Sigma-Aldrich), and added to $1.0 \mu \mathrm{l}$ oligo dT and $1.0 \mu \mathrm{l}$ dNTPs. This solution was denatured at $65^{\circ} \mathrm{C}$ for $5 \mathrm{~min}$, then incubated at $4^{\circ} \mathrm{C}$. It was then added to $1.0 \mu \mathrm{l}$ of $0.1 \mathrm{M}$ DTT, $4.0 \mu \mathrm{l} 5 \mathrm{X}$ buffer, $1.0 \mu 1$ SuperScript III Reverse Transcriptase (Invitrogen Life Technologies), and 1.0 $\mu 1$ Milli-Q water (Merck Millipore, Darmstadt, Germany) treated with DEPC. Samples were then incubated for $5 \mathrm{~min}$ at $25^{\circ} \mathrm{C}$, followed by one cycle of incubation at $50^{\circ} \mathrm{C}$ for $60 \mathrm{~min}$. Reactions were inactivated at $70^{\circ} \mathrm{C}$ for $15 \mathrm{~min}$, and the resulting cDNA was subjected to amplification with $2.5 \mu \mathrm{l} 10 \mathrm{X}$ buffer, $0.15 \mu \mathrm{l}$ dNTPs $(10 \mathrm{mM}), 0.75 \mu \mathrm{l} \mathrm{MgCl}_{2}$ (50 mM), $0.2 \mu \mathrm{l}$ Invitrogen Taq DNA polymerase, $1.0 \mathrm{ml}$ forward and reverse primers (each $10 \mu \mathrm{M}$ ), $200 \mathrm{ng}$ cDNA, and Milli-Q water treated with DEPC, to achieve a final volume of $25.0 \mu \mathrm{l}$ ).

Specific primers for $\beta$-actin and IL-12 were designed, as described by Tripathy et al (7). The following primers were used: Forward, CACTCTTCCAGCCTTCCTTCC and reverse, CGGACTCGTCATACTCCTGCTT for $\beta$-actin (annealing temperature, $64^{\circ} \mathrm{C}$; length, $311 \mathrm{bp}$ ); and forward, AGTGTCAAAAGCAGCAGAGG and reverse, AACGCAGAATGTCAGGGAG for IL-12 (annealing temperature, $66^{\circ} \mathrm{C}$; length, $363 \mathrm{bp}$ ). Amplification cycles were performed using a Thermocycler (Invitrogen Life Technologies), and included an initial denaturation at $94^{\circ} \mathrm{C}$ for $5 \mathrm{~min}$, followed by 40 cycles of $94^{\circ} \mathrm{C}$ for $40 \mathrm{sec}$, the annealing temperature specific to each primer for $45 \mathrm{sec}$ and an extension step at $72^{\circ} \mathrm{C}$ for $45 \mathrm{sec}$. A final incubation at $72^{\circ} \mathrm{C}$ for $10 \mathrm{~min}$ was followed by cooling samples to $16^{\circ} \mathrm{C}$. Amplified products were then subjected to electrophoresis on $10 \%$ polyacrylamide gels and stained with $2 \%$ silver nitrate. A Trackit ${ }^{\mathrm{TM}} 50$ bp DNA ladder (Invitrogen Life Technologies) provided molecular weight markers for comparison. As a control, and in order to verify the quality of the cDNA obtained, amplification of $\beta$-actin was performed for all samples analyzed.

Image acquisition and analysis. Following cell sorting, lymphocytes were added to each well of a 96-well black clear bottom microplate (Corning Inc., Corning, NY, USA) and centrifuged at $200 \mathrm{x} \mathrm{g}$ for $5-10 \mathrm{~min}$ at $4^{\circ} \mathrm{C}$. Images were 


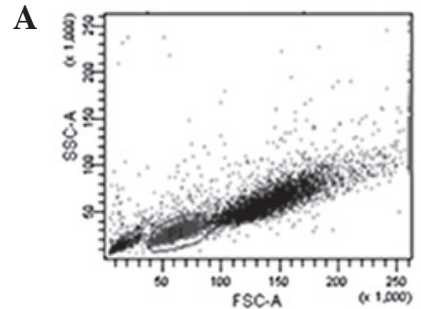

B
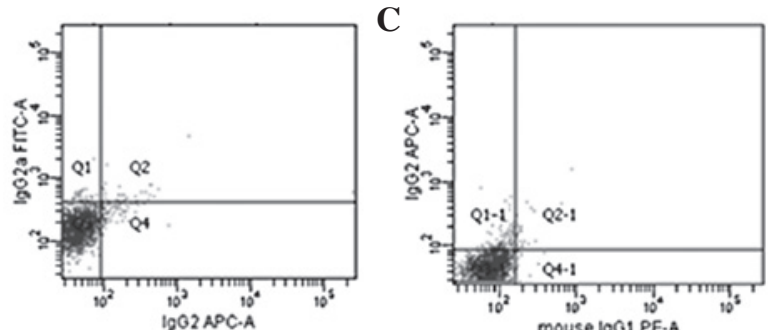

D
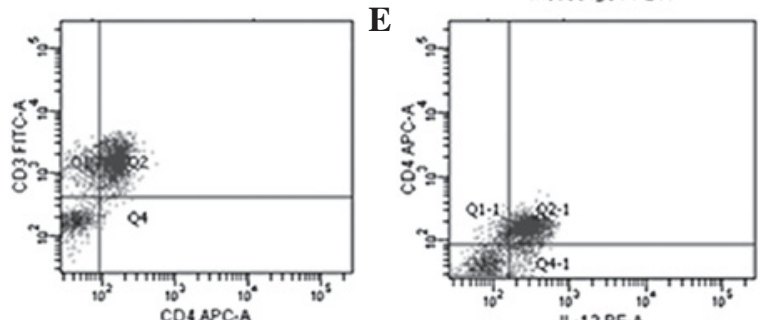

Figure 1. Identification of CD3+CD4+IL-12+ lymphocytes. Representative flow cytometry graphs, demonstrating positivity for CD3+CD4+IL-12+ lymphocytes from one patient. (A) Gating strategy for the identification of lymphocytes (P1). (B) and (C) control isotypes. (D) Double-positive CD3+CD4+ cells (Q2). (E) Double-positive CD4+IL-12+cells (Q2.1). IL-12, interleukin 12.

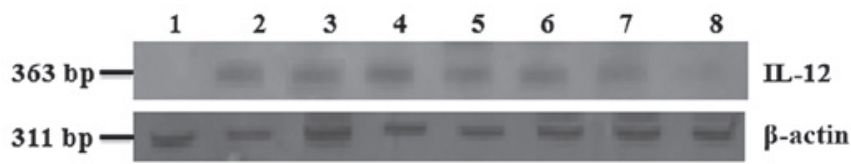

Figure 2. Reverse transcription-polymerase chain reaction products of CD3+CD4+IL-12+ cells, purified by cell sorting flow cytometry. Gel of electrophoresis demonstrating the expression of IL-12 and $\beta$-actin mRNA in $\mathrm{CD} 3+\mathrm{CD} 4+$ cells obtained from the $\mathrm{PB}$ of a healthy control (line 1) or patients with cancer (lines 2-8). IL-12, interleukin-12; PB, peripheral blood.

acquired using an automated microscope ImageXpress ${ }^{\circledR}$ Micro XL Widefield High-Content Screening System with a 40x Super Plan Fluor ELWD, NA 0.60 objective (Molecular Devices, Sunnyvale, CA, USA). The acquisition of multiple sites (25 to 36 sites) from each well, containing cells from healthy individuals or patients with cancer, was performed for each fluorescent probe using Deconvolution Wiener Filter K, Value $=0.2$. Images were captures using MetaXpress ${ }^{\circledR}$ 5.3.0.1 Software (Molecular Devices).

\section{Results}

According to the flow cytometry results, the percentage of IL-12+ cells among the CD3+CD4+ cell population obtained from cancer patients ranged from 2.2-75.4\% (median, 25.7\%). The intensity of IL-12 fluorescence, assessed by flow cytomtery, ranged from 168.0 to 2,371.0 (median, 317.5). By contrast,
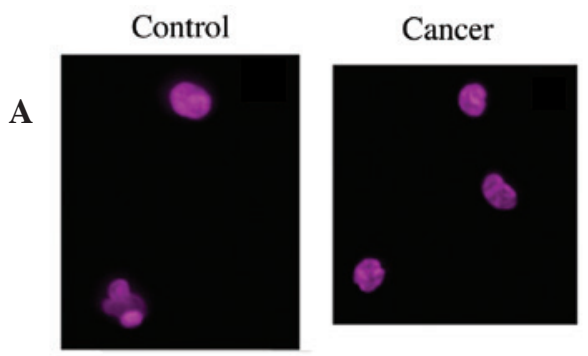

B
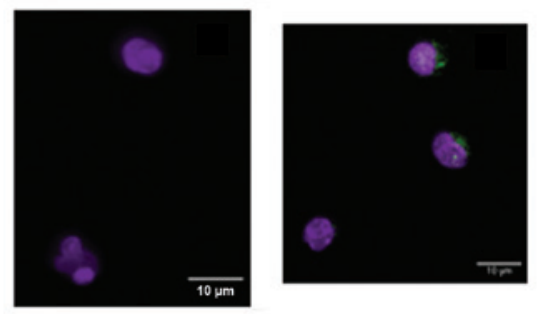

C
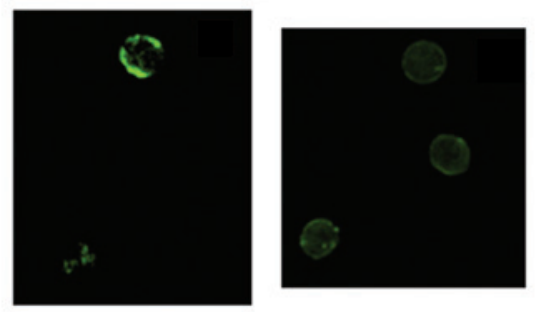

D
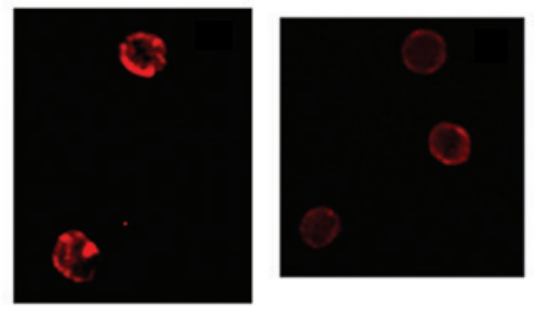

$\mathbf{E}$
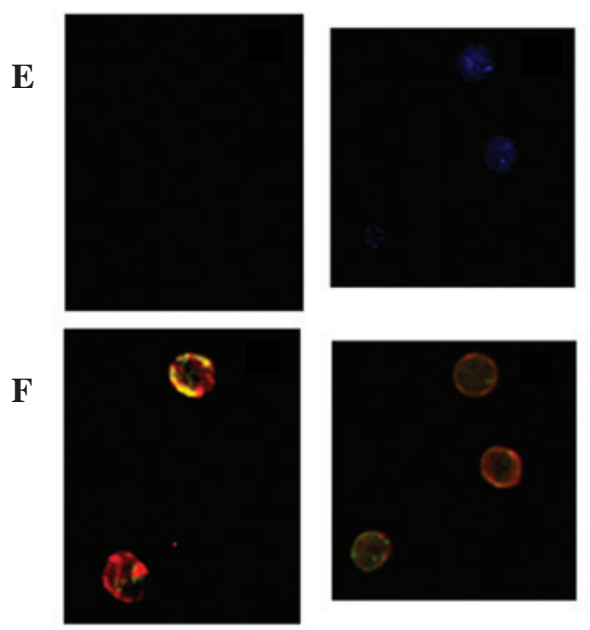

$\mathbf{G}$
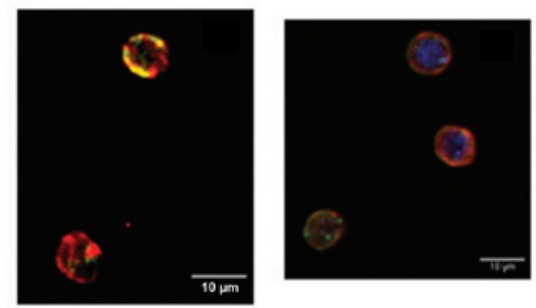

Figure 3. Intracytoplasmatic localization of IL-12 in helper T lymphocytes. Fluorescent image acquisition of peripheral blood cells from healthy controls (left) and patients with cancer (right), with (A) fluorescent nuclear staining and nuclear staining in addition to (B) $\alpha$-IL-12 PE, (C) $\alpha$-CD3 FITC, (D) $\alpha$-CD4 APC, (E) $\alpha$-IL-12 PE, (F) $\alpha$-CD3 FITC and $\alpha$-CD4 APC, or (G) $\alpha$-CD3 FITC, $\alpha$-CD4 APC and $\alpha$-IL-12 PE. Magnification, x40. IL-12, interleukin-12; PE, phycoerythrin; FITC, fluorescein isothiocyanate; APC, allophycocyanin. 
cells obtained from healthy controls in the same conditions and using the same antibodies did not exhibit basal IL-12 synthesis in CD3+CD4+ cells. Control isotypes were used in all samples studied.

The cells identified by flow cytometry in cancer patients as CD3+CD4+IL-12+ (Fig. 1) were separated by cell sorting and, using specific primers for IL-12, subjected to RT-PCR. The cells obtained from all cancer patients expressed mRNA for IL-12. In control patients, cell sorting was used to isolate CD3+CD4+ cells identified by flow cytometry and, using the same primers for IL-12, subjected to RT-PCR. $\beta$-actin was used as a control and was present in all patient and control samples studied. The results are presented in Fig. 2.

The presence of intracytoplasmatic IL-12 in CD3+CD4+ lymphocytes (measuring $\sim 10 \mu \mathrm{m}$ in diameter) is shown in Fig. 3.

\section{Discussion}

The anticancer immune response in a number of types of cancer is mediated by IL-12 (8), which may therefore be a candidate for use in tumor immunotherapy, due to its capacity to active the innate and adaptive immune responses (9).

IL-12 is important in the immune response of patients with cancer, who are treated with immunotherapy, as the use of various approaches used to stimulate the antitumoral immune response depends on an increase in the expression of this mediator. Patients with advanced cancer, who were administered a DC vaccine, exhibited increased levels of IL-12 during treatment (5). Patients with grade II or III cervical intraepithelial neoplasia (CIN) also exhibited increased levels of IL-12 following intrastromal cervical injection of interferon $\alpha 2 b$. This increase was primarily observed in patients that present CIN regression in response to this immunotherapy (10). In mice, cytotoxic $\mathrm{T}$ lymphocytes that have been genetically modified to produce IL-12, have been shown to infiltrate and potentially reduce the size of tumors (11).

The present study was conducted in patients with cancer, who had either not received treatment, or were $\geq 2$ months post-treatment. Regardless of the initial location (endometrium, uterine cervix and others) and stage of neoplasia, all patients exhibited detectable IL-12 mRNA in PB CD4+ T lymphocytes.

The current study raises the question of which immunological conditions or diseases induce IL-12 production by PB CD4+ T Lymphocytes. Notably, one of the cases analyzed, although not included in the present study, initially received surgery for presumed ovarian cancer. However, following pathological analysis, the patient was diagnosed with disseminated abdominal tuberculosis. This patient also exhibited IL-12 mRNA and protein expression in intracellular CD4+ lymphocytes. As cancer and tuberculosis are two chronic diseases, it is hypothesized that IL-12 production by helper T lymphocytes occurs during chronic stimulation of the immune system. However, further research in other diseases is required in order to confirm or refute this hypothesis, and to improve understanding of the function of IL-12 in helper T lymphocytes.

In accordance with the results of the present study, Kuka et al (12) described $\alpha \beta T C R+$ cells that expressed CD11c, MHC class II and IL-12 in mouse splenocytes and human peripheral blood mononuclear cells, which had been stimulated with polyclonal activators. The primary difference between the results of this study and the current study, are that the latter used RT-PCR to demonstrate that the cells produced IL-12 mRNA spontaneously, and did not require stimulation to synthesize this cytokine. It should be noted that the cells in the present study may be different from those described by Kuka et al, as the preliminary results from the current study demonstrated that the cells were CD11c- and MHC II-negative. Furthermore, the present study demonstrated by images that the cells described have the same measurement as lymphocytes and not dendritic cells.

In conclusion, the current results showed that a percentage of CD4+ T lymphocytes in the PB of patients with cancer produce IL-12 mRNA, as demonstrated by RT-PCR, and that this cytokine is present in the cytoplasm of the cells. Further investigation in acute and chronic diseases is required in order to determine the conditions in which IL-12 is produced and secreted, in addition to elucidating the function of IL-12 production by PB CD4+ lymphocytes.

\section{Acknowledgements}

The authors would like to thank the Studies and Projects Funding Body (FINEP), the Foundation for Research Assistance of the State of Minas Gerais (FAPEMIG), the National Council for Scientific and Technical Development (CNPq), the Uberaba Foundation for Teaching and Research (FUNEPU) for financial assistance and Dr Luciane Ganiko (Molecular Devices Brazil) for assistance with cell imaging.

\section{References}

1. Mosmann TR and Coffman RL: Th1 and Th2 cells: Different patterns of lymphokine secretion lead to different functional properties. Annu Rev Immunol 7: 145-173, 1989.

2. Murphy KM and Stockinger B: Effector T cell plasticity: Flexibility in the face of changing circumstances. Nat Immunol 11: 674-680, 2010

3. Vignali DA and Kuchroo VK: IL-12 family cytokines: Immunological playmakers. Nat Immunol 13: 722-728, 2013.

4. Wang J, Nong L, Wei Y, Qin S, Zhou Y and Tang Y: Association of interleukin-12 polymorphisms and serum IL-12 p40 levels with osteosarcoma risk. DNA Cell Biol 32: 605-610, 2013.

5. Rodrigues CM, Matias BF, Murta EF and Michelin MA: The role of T lymphocytes in cancer patients undergoing immunotherapy with autologous dendritic cells. Clin Med Insights Oncol 5: 107-115, 2011.

6. Michelin MA, Abdalla DR, Aleixo AA and Murta EF: Peripheral helper lymphocytes produce interleukin 12 in cancer patients. Clin Med Insights Oncol 7: 75-81, 2013.

7. Tripathy NK, Chauhan SK and Nityanand S: Cytokine mRNA repertoire of peripheral blood mononuclear cells in Takayasu's arteritis. Clin Exp Immunol 138: 369-374, 2004.

8. Jones LL, Chaturvedi V,Uyttenhove C, Van Snick J and Vignali DA: Distinct subunit pairing criteria within the heterodimeric IL-12 cytokine family. Mol Immunol 51: 234-244, 2012.

9. Lasek W, Zagożdżon R and Jakobisiak M: Interleukin 12: Still a promising candidate for tumor immunotherapy? Cancer Immunol Immunother 63: 419-435, 2014.

10. Misson DR, Abdalla DR, Borges AM, Shimba DS, Adad SJ, Michelin MA and Murta EF: Cytokine serum levels in patients with cervical intraepithelial neoplasia grade II-III treated with intralesional interferon- $\alpha$ 2b. Tumori 97: 578-584, 2011.

11. Kerkar SP, Muranski P, Kaiser A, Boni A, Sanchez-Perez L, Yu Z, Palmer DC, Reger RN, Borman ZA, Zhang L, et al: Tumor-specific CD8+ T cells expressing IL-12 eradicate established cancers in lymphodepleted hosts. Cancer Res 70: 6725-6734, 2010.

12. Kuka M, Munitic I and Ashwell JD: Identification and characterization of polyclonal $\alpha \beta$ T cells with dendritic cell properties. Nat Commun 3: 1223, 2012. 